\title{
Research on Relationship between Curvature and Torsion of Space Curve
}

\author{
Yong Huang ${ }^{1, a}$ \\ ${ }^{1}$ College of Mathematics and Statistics, Zhaotong University, Zhaotong 657000, China \\ ${ }^{\mathrm{a}}$ email
}

Keywords: Curvature, Torsion, Space Curve

\begin{abstract}
The space curve of curvature, torsion and formula is a part of the basic theory of space curve, it is close to plane space curve and prism of three basic knowledge as a basis. The space curve of curvature, torsion and formula in the basic theory of space curve occupies an important position, is some basic properties and the basic equation of space curve. The curve of curvature and torsion completely determines the shape of the curve. This paper from the definition, formula and curvature, to illustrate the three aspects gradually analytic space curves of torsion.
\end{abstract}

\section{Introduction}

Differential geometry teaching requires students to make full use of the analytic geometry, calculus, linear algebra, differential equations and other professional basic courses of basic knowledge and basic thinking method to learn the basic concepts, the basic theory of local differential geometry, can fully reflect the value prerequisite foundation course, can effectively stimulate students on the basis of professional knowledge and understanding then, consolidate the foundation to promote students to strengthen professional training, spatial imagination ability and spatial analysis ability and expression ability, improve the ability of logical reasoning, training theory and practice of analysis and use of mathematical knowledge to solve practical problems, and prepare for the further professional. Therefore, teachers should not only by the final standard examination scores to measure the level of student ability, but according to the teaching content, innovative teaching methods, the actual level of diversification of evaluation of students, and with the development of the perspective of the students, students learn to appreciate, to provide more space for students in self play. Therefore, teachers should adopt flexible and effective way, through a variety of ways, to mobilize students' active participation in class discussion and study, so as to fully stimulate the main role of the students, learning initiative learning. In recent years, the course of differential geometry teachers gradually tried various teaching methods, the timely set group activities appropriate to active learning atmosphere, especially recently by groups in Waterloo mathematics competition match part of the race inspired by a team relay race, obviously led to more different levels of ability of students into the classroom learning process. This method pay attention to using a variety of teaching methods and assessment methods, "pay attention to the cultivation of students' self-efficacy, pay attention to strengthening students' self-exploration and practice in learning, so that students need to strengthen learning to recognize them.

\section{Curvature of Space Curve}

We first study the concept of curvature of space curve. The curve of different points in different or the same curve, degree of bending of the curve may be different. For example, the larger radius circular bending degree is smaller, and the smaller radius of circular bending greatly. In order to describe accurately the curvature of the curve, we introduce the concept of curvature.

The exact definition leads to curvature from the intuitive basis, we first note that the greater the degree of bending curve, from point to point changes, the faster change of its tangent vector direction. So as the curve of average degree of bending in the known segment for the desirable curve in the tangent vector on average between the rotation angle of the arc long. 
The equation of the class curve in the space is $r=r(s)$.

A point on the curve, the natural parameters, the other adjacent points, the natural parameters for each curve. The unit tangent vector $\boldsymbol{\alpha}(s)$ and the tangent vector angle $\boldsymbol{\alpha}(s+\Delta s)$. It is the tangent vector to the translation, and the angle between two vectors is $\Delta \varphi$.

We define the curvature of the curve at the point of rotation of the length of the tangent vector at the tangent of the space curve.

Definition: The curvature of the space curve at the point of $p$ is

$$
k(s)=\lim _{\Delta s \rightarrow 0}\left|\frac{\Delta \varphi}{\Delta s}\right|
$$

$\Delta s$ is the arc length between points and their adjacent points; $\Delta \varphi$ is the angle between the tangent vector of the point and the sum of the curve.

From the definition of the curvature of space curve can be seen, its geometric significance is tangent vector of a curve for the rotational speed of the arc length. When the curve in a greater degree of bending, tangent vector rotation speed for arc length is greater, so the curvature curve depicts the bending degree. Since there are many curvatures on the surface, it is an important subject to study these curvatures and the relations between them, and they are important representations of geometric properties on surfaces. This leads to a class of questions that characterize the geometric properties of surfaces with various curvatures and their relations. This type of solution is obtained. Basic quantities by vector surface equation, and use various relation formula between them to solve related problems. Different kinds of curve surface must study and the relationship between them, a variety of coordinate net and its geometric properties in the net of the curve. Now, differential geometry has become the institutions of higher mathematics and applied mathematics is an important course of the course by means of geometric vector calculus and space coordinate frame, the geometric problem to solve all kinds of space curves and surfaces. Because it has very strong theory and abstract symbols, plus multi formula, which can be cited a few examples of intuitive, weak, so many students in the study of differential geometry, often feel difficult to do, especially difficult to prove that, in any discipline, has its own laws, differential geometry there is no exception in this paper, the differential geometry learning some advice.

For a space curve, the curve is not only bent but also twisted (leaving the close plane), so it is not enough to study the curvature of the space curve, but also to describe the degree of torsion of the degree of the curve twist

\section{Torsion of Space Curve}

When the curves of torsion, binormal vector (or close plane) with the change of position (Figure 1), so we use the binormal vector (or close plane) to characterize the torsional degrees of rotational speed curve (in a left plane close degree).

Now the natural parameter of the point on the curve is given, the parameter of the other point is the secondary normal vector of the curve at two points, and the angle between the two pairs of normal vectors $\gamma(s)$ and $\gamma(s+\Delta s)$ is $\Delta \varphi$. 


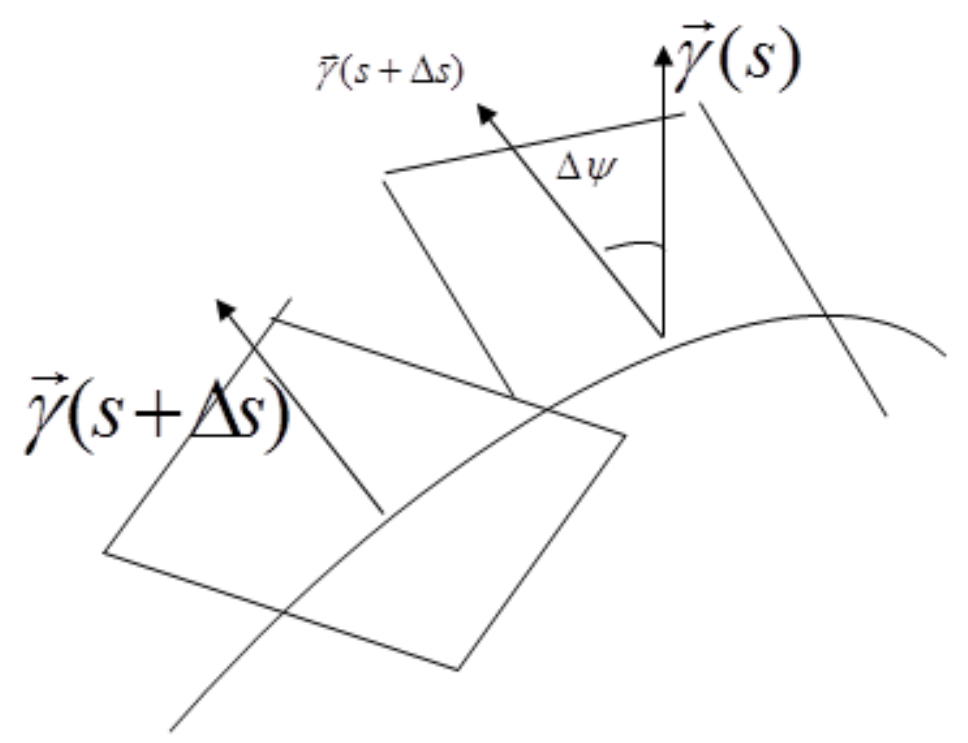

Figure 1. Figure of torsion of space curve

Definition: The curvature of the space curve $(c)$ in p point is:

$$
\tau(s)=\frac{(\dot{\vec{r}}(s), \ddot{\vec{r}}(s), \ddot{\vec{r}}(s))}{(\ddot{\vec{r}}(s))^{2}} .
$$

\section{Relationship between Curvature and Torsion of Space Curve}

If $r=r(s)$, we have:

$$
\begin{gathered}
r^{\prime}=\frac{d r}{d s} \frac{d s}{d t}=\dot{r} \frac{d s}{d t} \\
r^{\prime \prime}=(\dot{r})^{\prime} \frac{d s}{d t}+\dot{r} \frac{d^{2} s}{d t^{2}}=\frac{d \dot{r}}{d s}\left(\frac{d s}{d t}\right)^{2}+\dot{r} \frac{d^{2} s}{d t^{2}}=\dot{r}\left(\frac{d s}{d t}\right)^{2}+\dot{r} \frac{d^{2} s}{d t^{2}}
\end{gathered}
$$

Therefore,

$$
r^{\prime} \times r^{\prime \prime}=\dot{r} \frac{d s}{d t} \times\left[\ddot{r}\left(\frac{d s}{d t}\right)^{2}+\dot{r} \frac{d^{2} s}{d t^{2}}\right]=\dot{r} \times \ddot{r}\left(\frac{d s}{d t}\right)^{3}
$$

So,

$$
\left|r^{\prime} \times r^{\prime \prime}\right|=|\cdot| \cdot|\cdot \cdot|\left(\frac{d s}{d t}\right)^{3} \sin \theta
$$

As

$$
|\dot{r}|=1, \dot{r} \perp \ddot{r}, \frac{d s}{d t}=\left|r^{\prime}\right|
$$

We have

$$
k=\frac{\left|r^{\prime} \times r^{\prime \prime}\right|}{\left|r^{\prime}\right|^{3}}
$$

The absolute value of the torsion measures the change rate of the angle between the angles of the normal vectors near the two points on the curve. A plane curve is a curve whose torsion constant is zero. If the space curve does not fall on a plane, it is called a deflection curve. 
In the basic differential geometry of a three dimensional curve, the torsion of a curve represents the degree of distortion of the curvature plane. In a word, the curvature and torsion of the space curve are similar to the curvature of the plane curve. For example, they are the coefficients of the differential equation system of the Frenet framework given by the Frenet-Serret formula.

The absolute value of the torsion measures the change rate of the angle between the angles of the normal vectors near the two points on the curve. A plane curve is a curve whose torsion constant is zero. If the space curve does not fall on a plane, it is called a deflection curve.

In the basic differential geometry of a three dimensional curve, the torsion of a curve represents the degree of distortion of the curvature plane. In a word, the curvature and torsion of the space curve are similar to the curvature of the plane curve. For example, they are the coefficients of the differential equation system of the Frenet framework given by the Frenet-Serret formula.

Curvature is bending, torsion is distortion. On a plane curve, the main vector is in the plane and vertical tangent vector. A method of vector cut is equal to the vector product method vector, and perpendicular to the plane. Since the secondary normal vector of plane curve is perpendicular to the plane, the torsion of plane curve is zero everywhere. That is, bending, not twisting. For 3D curves, the curvature of a point is not zero, and bending and twisting occur simultaneously. It's about the torsion of a curve in a three-dimensional space. The curvature and torsion of surface can be calculated by analogy. For higher dimensional torsion (including curvature), differential geometry is used.

Curvature is a measure of the degree of roughness of a body. Flatness has different meanings to different geometry.

In this paper, the curvature of curves and surfaces in Euclidean space is considered. In general, the curvature tensor is referred to.

In dynamics, curvature is produced when a body moves in speed with respect to another object. It's about distortions of time and space. According to the equivalent principle of general relativity, a moving object can be regarded as a gravitational field, thus generating curvature.

According to general relativity, in the gravitational field, the spatial and temporal properties of objects is determined by the "quality" of the distribution, the distribution of objects in the "quality" of the spatio-temporal properties are not uniform, the space-time bending caused. Because an object has mass, it will bend time and space, and you can think that with speed, mass objects become heavier, curvature of space and time curvature is even greater.

\section{Acknowledgement}

The paper was financially supported by Scientific Research Foundation of Education Department of Yunnan Province (Grant No. 2014Y499).

\section{References}

[1] Lu Yazhe, Hu Yan, Huang Weihua. Geodesic Curvature and Geodesic Torsion on the Helicoid [J]. Journal of Lanzhou Universit y of Arts and Science (Natural Sciences), 2015, 29(6): 26-28.

[2] Guo Zhanhai. Conclusions on the Vertical Curvature and Torsion of the Corresponding Tangent of Two Curves [J]. Journal of Hetao College, 2017, 14(2): 85-88.

[3] Pan Quanxiang. Curvature,torsion and Frenet formulas for a regular curve and its application [J]. Journal of Henan Institute of Science and Technology, 2012, 41(3): 69-71+76.

[4] Huang Rui, Chu Yawei. The Calculation of the Curvature and the Torsion of the Curve of Implicit Function Form [J]. Journal of Huaibei Normal University (Natural Science ), 2016,37(1): 72-74. 